OPEN ACCESS

Edited by: Maria J. Delgado,

Estación Experimental del Zaidín (CSIC), Spain

Reviewed by:

Emilio Fernandez,

Universidad de Córdoba, Spain

Patricia Coutinho Dos Santos, Wake Forest University, United States

${ }^{*}$ Correspondence:

Luis M. Rubio

Im.rubio@upm.es

Specialty section:

This article was submitted to Terrestrial Microbiology, a section of the journal

Frontiers in Microbiology

Received: 14 January 2019

Accepted: 06 March 2019

Published: 21 March 2019

Citation:

Navarro-Rodríguez M, Buesa JM and Rubio LM (2019) Genetic and

Biochemical Analysis of the Azotobacter vinelandii Molybdenum

Storage Protein.

Front. Microbiol. 10:579.

doi: 10.3389/fmicb.2019.00579

\section{Genetic and Biochemical Analysis of the Azotobacter vinelandii Molybdenum Storage Protein}

\author{
Mónica Navarro-Rodríguez, José María Buesa and Luis M. Rubio* \\ Centro de Biotecnología y Genómica de Plantas (CBGP), Universidad Politécnica de Madrid (UPM), Instituto Nacional de \\ Investigación y Tecnología Agraria y Alimentaria (INIA), Madrid, Spain
}

The $\mathrm{N}_{2}$ fixing bacterium Azotobacter vinelandii carries a molybdenum storage protein, referred to as MoSto, able to bind 25-fold more Mo than needed for maximum activity of its Mo nitrogenase. Here we have investigated a plausible role of MoSto as obligate intermediate in the pathway that provides Mo for the biosynthesis of nitrogenase iron-molybdenum cofactor (FeMo-co). The in vitro FeMo-co synthesis and insertion assay demonstrated that purified MoSto functions as Mo donor and that direct interaction with FeMo-co biosynthetic proteins stimulated Mo donation. The phenotype of an $A$. vinelandii strain lacking the MoSto subunit genes $(\triangle m o s A B)$ was analyzed. Consistent with its role as storage protein, the $\triangle m o s A B$ strain showed severe impairment to accumulate intracellular Mo and lower resilience than wild type to Mo starvation as demonstrated by decreased in vivo nitrogenase activity and competitive growth index. In addition, it was more sensitive than the wild type to diazotrophic growth inhibition by $\mathrm{W}$. The $\triangle$ mosAB strain was found to readily derepress vnfDGK upon Mo step down, in contrast to the wild type that derepressed Vnf proteins only after prolonged Mo starvation. The $\triangle \operatorname{mos} A B$ mutation was then introduced in a strain lacking $\vee$ and Fe-only nitrogenase structural genes ( $\Delta v n f \Delta a n f)$ to investigate possible compensations from these alternative systems. When grown in Mo-depleted medium, the $\triangle \operatorname{mos} A B$ and $\operatorname{mos} A B^{+}$strains showed low but similar nitrogenase activities regardless of the presence of $\mathrm{Vnf}$ proteins. This study highlights the selective advantage that MoSto confers to $A$. vinelandii in situations of metal limitation as those found in many soil ecosystems. Such a favorable trait should be included in the gene complement of future nitrogen fixing plants.

Keywords: nitrogenase, iron-molybdenum cofactor, nitrogen fixation, metal homeostasis, MoSto

\section{INTRODUCTION}

Nitrogenase, the enzyme complex that catalyzes the fixation of $\mathrm{N}_{2}$ into $\mathrm{NH}_{3}$, is one of the most relevant enzymes in the nitrogen cycle since it converts inert $\mathrm{N}$ into a biologically usable form. In its most prevalent type nitrogenase is an iron-sulfur molybdoenzyme (Boyd and Peters, 2013), although other phylogenetically related nitrogenases exist that carry iron-sulfur-vanadium or ironsulfur only cofactors (Bishop and Joerger, 1990; Eady, 1996). The Mo-nitrogenase consists of a dinitrogenase component, a NifDK heterotetramer containing two pairs of metalloclusters named 
P-cluster (8Fe-7S) and FeMo-co (7Fe-9S-C-Mo-R-homocitrate) (Kim and Rees, 1992; Einsle et al., 2002; Rubio and Ludden, 2008; Spatzal et al., 2011), and a dinitrogenase reductase component formed by two NifH homodimers each one carrying a [4Fe4 S] cluster (Georgiadis et al., 1992). Azotobacter vinelandii has the peculiarity of having genes to encode the Mo-nitrogenase (nif) and the alternative $\mathrm{V}(v n f)$ and Fe-only (anf) nitrogenases (Bishop and Joerger, 1990). The dinitrogenase components of the alternative nitrogenases contain additional subunits (VnfG or AnfG) essential for $\mathrm{N}_{2}$ reduction (Chatterjee et al., 1997; Krahn et al., 2002) and present subtle differences in cofactor structure (Sippel and Einsle, 2017). However, amino acid sequence comparisons of NifD/VnfD/AnfD and NifK/VnfK/AnfK indicate that residues that serve as ligands to the metal cofactors are conserved in all three nitrogenases (Joerger et al., 1990).

Azotobacter vinelandii nif, vnf, and anf genes are differentially expressed depending on the metal availability in the environment following a hierarchical nif $>v n f>$ anf sequence. The presence of as low as $50 \mathrm{nM}$ molybdate in the medium represses $v n f$ and anf genes while vanadium represses the anf but not the nif genes (Jacobson et al., 1986; Luque and Pau, 1991; Jacobitz and Bishop, 1992). As consequence V-nitrogenase is active in the absence of Mo when $\mathrm{V}$ is available and Fe-only nitrogenase is active when neither Mo nor $\mathrm{V}$ is available in the medium.

Molybdenum is an essential transition metal for most organisms, which typically carry a number of enzymes and proteins involved in its uptake, storage, homeostasis, regulation, and Mo cofactor biosynthesis (Hernandez et al., 2009; Hille et al., 2014). This metal, and its biological antagonist tungsten (W), can exist in several oxidation states ranging from - II to $+\mathrm{VI}$, with $\mathrm{MoO}_{4}{ }^{2-}$ being the main source of $\mathrm{Mo}$ at neutral and basic $\mathrm{pH}$. However, Mo availability in soil ecosystems depends on $\mathrm{pH}$, reactive oxides and water drainage and it is often a limiting factor for nitrogen fixation (Reddy et al., 1997).

Bacteria have developed high-affinity chelation and uptake mechanisms to scavenge molybdate from the environment, including metallophores, ABC-type transporters, and Mo storage proteins (Mouncey et al., 1995; Kraepiel et al., 2009). A. vinelandii carries an unusual Mo-binding protein called molybdenumstorage protein (MoSto) (Pienkos and Brill, 1981; Fenske et al., 2005), a $(\alpha \beta)_{3}$ hexameric complex encoded by the homologous mos $A$ and mos $B$ genes that is capable of binding more than 100 Mo atoms in the form of polyoxomolybdate clusters (Schemberg et al., 2007; Kowalewski et al., 2012; Poppe et al., 2014). MoSto can also accumulate W (Schemberg et al., 2007). Metal binding to MoSto requires ATP hydrolysis while metal release is ATPindependent but $\mathrm{pH}$-dependent occurring stepwise above $\mathrm{pH} 6.8$ (Schemberg et al., 2008). No amino acid sequence to other Mocontaining enzymes has yet been described. MoSto structure has been related to nucleoside monophosphate kinases, particularly with the UMP kinase from bacteria and archaea, which uses ATP to phosphorylate UMP (Ramon-Maiques et al., 2002; Poppe et al., 2014).

Azotobacter vinelandii is known to accumulate 25 -fold more Mo than required for maximum nitrogenase activity (Shah et al., 1984) probably due to the presence of MoSto. However, the effect on nitrogenase of eliminating MoSto has never been determined.
The aims of this study are the phenotypical characterization of a MoSto deficient strain and the elucidation of MoSto involvement in FeMo-co biosynthesis.

\section{RESULTS}

\section{MoSto Serves as Mo Donor for in vitro FeMo-co Biosynthesis}

Two versions of MoSto were purified to test their capacity to serve as Mo donor in the in vitro FeMo-co synthesis assay. A non-tagged version was partially purified from cells of A. vinelandii DJ (Figure 1A) whereas a histidine-tagged version (hereinafter named rMoSto) was cloned, overexpressed, and purified from recombinant Escherichia coli cells (Figure 1B). As purified from A. vinelandii, MoSto carried 25-fold more Mo than rMoSto purified from E. coli cells grown in medium supplemented with $1 \mathrm{mM}$ molybdate (Table 1). When purified from cells grown in not supplemented medium, rMoSto contained very little Mo, consistent with BL21 deficiency in high-affinity molybdate transport (Pinske et al., 2011). Both MoSto and rMoSto functioned as sole Mo source for in vitro FeMo-co synthesis, with both versions being equally efficient when normalized by Mo content (Table 1). Nitrogenase reconstitution levels correlated with Mo contents in all MoSto preparations. Direct interaction with FeMoco biosynthetic proteins stimulated Mo donation by MoSto (Figure 1C). When MoSto (or rMoSto) was separated from the Nif proteins by a dialysis membrane threefold lower nitrogenase reconstitution levels were obtained (Figure 1D). These basal levels of reconstitution were probably due to Mo release from MoSto at $\mathrm{pH} 7.5$ of the reaction mixture (Schemberg et al., 2008). Interestingly, reconstitution due to unspecific Mo release did not increase over time. These biochemical assays established a role for MoSto as direct Mo donor for FeMo-co synthesis.

\section{The Absence of MoSto Impairs Molybdenum Accumulation and in vivo Nitrogenase Activity}

An $A$. vinelandii strain with an in-frame deletion of MoStoencoding genes $(\triangle m o s A B)$ was generated to investigate cellular molybdenum levels and nitrogenase activity dependence on MoSto. Four culture conditions reflecting different levels and lengths of Mo starvation were tested. On one hand, precultures obtained by subculturing at least three times in Mo-limited Burk's modified medium (hereinafter called Mo Starved) were inoculated into N-free Mo-limited medium (-Mo) or into N-free Mo-standard medium (+Mo) (Figure 2A). On the other hand, precultures grown in Burk's modified medium (hereinafter called Mo Standard) were inoculated into $\mathrm{N}$-free Mo-limited medium (-Mo) or into N-free Mo-standard medium (+Mo) (Figure 2B). To minimize nitrogen stress all testing growth media contained $1 \mu \mathrm{M}$ vanadate to allow $\mathrm{V}$-nitrogenase synthesis at low Mo concentrations. 
A

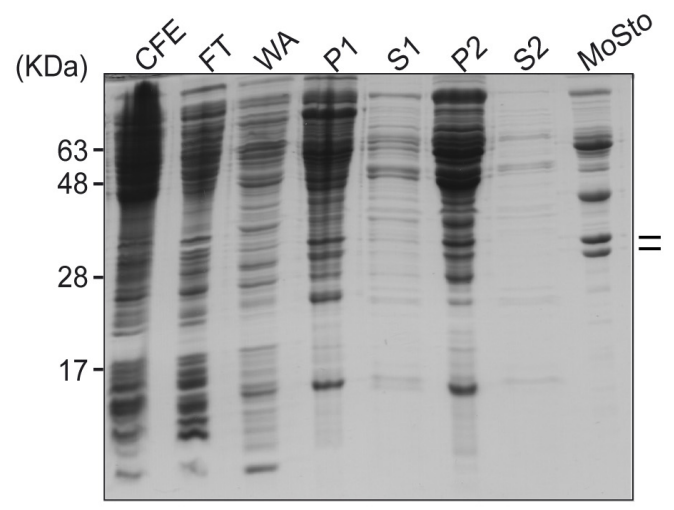

C

Mo source together with Nase components

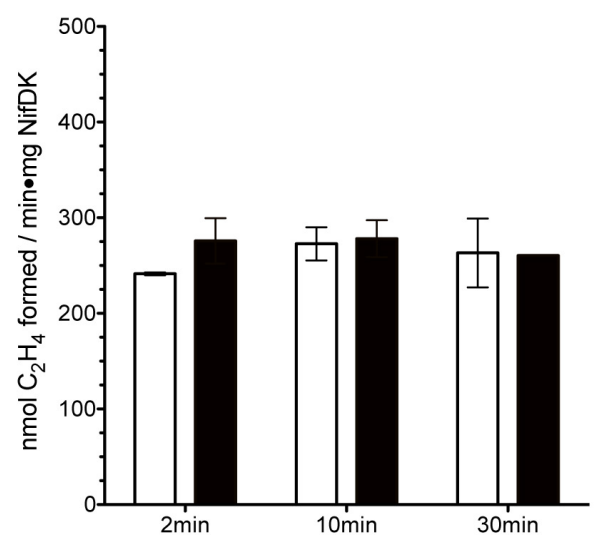

B

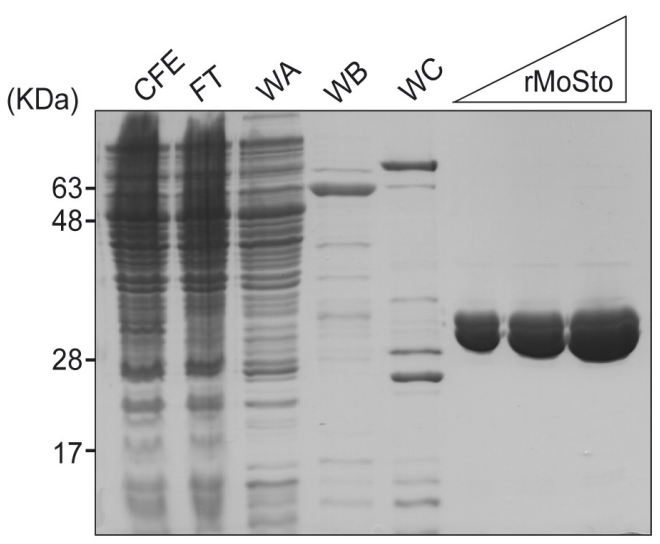

D

Mo source separated from Nase components

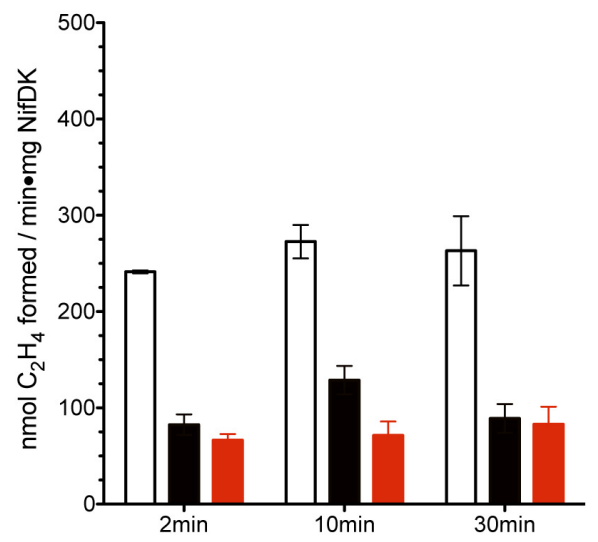

FIGURE 1 | In vitro FeMo-co synthesis and nitrogenase reconstitution time-course assays using MoSto as source of Mo. (A) Partial purification of MoSto from Azotobacter vinelandii cells. Lines indicate migration of MoSto subunits. (B) Purification of rMoSto from Escherichia coli cells. CFE, cell-free extract; FT, first column flow through; WA, WB, and WC column washes with increasing imidazole; P and S, pellet and supernatant of ammonium sulfate fractionations. (C) Reactions in which the molybdenum source (molybdate or MoSto) is mixed with the FeMo-co biosynthetic proteins. Molybdenum sources used were: $7.5 \mu \mathrm{M}$ molybdate (white bars) or MoSto protein equivalent to $4.5 \mu \mathrm{M} \mathrm{Mo} \mathrm{(black} \mathrm{bars).} \mathrm{(D)} \mathrm{Reaction} \mathrm{mixtures} \mathrm{in} \mathrm{which} \mathrm{the} \mathrm{molybdenum} \mathrm{source:} \mathrm{molybdate} \mathrm{(white} \mathrm{bars),} \mathrm{MoSto} \mathrm{(black} \mathrm{bars),} \mathrm{or}$ rMoSto (red bars) is separated from FeMo-co biosynthetic proteins by a dialysis membrane. Molybdenum sources used were: $7.5 \mu \mathrm{M}$ molybdate, $22.8 \mu \mathrm{g}$ MoSto (4.5 $\mu \mathrm{M}$ Mo), or $229 \mu \mathrm{g}$ rMoSto (11 $\mu \mathrm{M}$ Mo). Data represent mean \pm standard deviation of three independent experiments.

TABLE 1 | Activity of nitrogenase reconstituted with MoSto as Mo donor in the in vitro FeMo-co synthesis assay.

\begin{tabular}{|c|c|c|c|c|}
\hline MoSto type & $\begin{array}{l}\text { Mo in growth } \\
\text { medium }(\mathrm{mM})^{\mathrm{a}}\end{array}$ & Mo atoms in MoSto ${ }^{b}$ & $\begin{array}{c}\mu \mathrm{g} \text { MoSto used ( } \mu \mathrm{M} \text { Mo in } \\
\text { reaction) }\end{array}$ & $\%$ Activity $^{\mathrm{c}}$ \\
\hline \multirow[t]{2}{*}{ rMoSto } & - & $0.05 \pm 0.00$ & $10(<0.01)$ & $2.3 \pm 0.8$ \\
\hline & & & $40(0.02)$ & $4.5 \pm 0.3$ \\
\hline \multirow[t]{2}{*}{ rMoSto } & 1 & $4.12 \pm 1.18$ & $10(0.48)$ & $34.3 \pm 3.2$ \\
\hline & & & $40(1.94)$ & $78.7 \pm 4.6$ \\
\hline \multirow[t]{2}{*}{ MoSto } & 0.01 & $104.14 \pm 19.8$ & 0.5 (0.99) & $55.3 \pm 4.9$ \\
\hline & & & $1.0(1.98)$ & $63.1 \pm 6.3$ \\
\hline
\end{tabular}

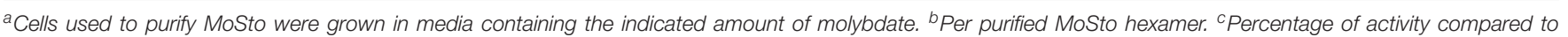

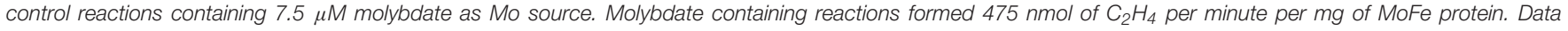
represent mean and standard deviations of three independent experiments.

The following observations could be made: $(i)$ In the presence of MoSto, Mo uptake by Mo-starved cells occurred much faster and maximum Mo accumulation was 100-fold higher; (ii) Mo content in cells lacking MoSto did not always correlate with nitrogenase levels (Figures 2A,B) suggesting the presence of alternative Mo sinks or reservoirs; (iii) MoSto was not 


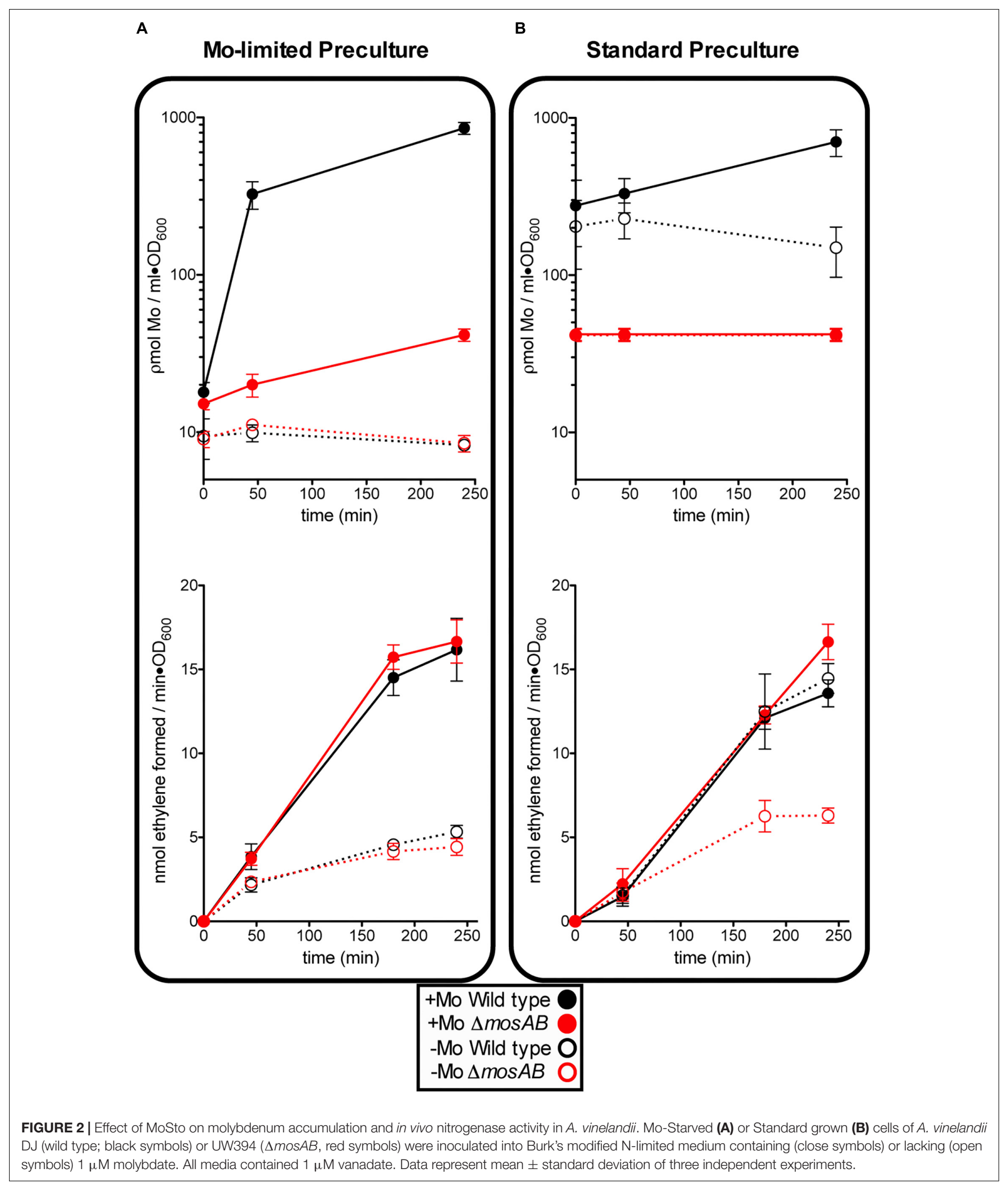

required for maximum nitrogenase activity as long as Mo was provided in the growth medium indicating that its role in Mo-nitrogenase is not essential; (iv) However, under transient
Mo starvation, tested by derepressing standard precultures in Mo-limited medium, the MoSto mutant was impaired in nitrogenase activity while the wild type was not (compare 
A

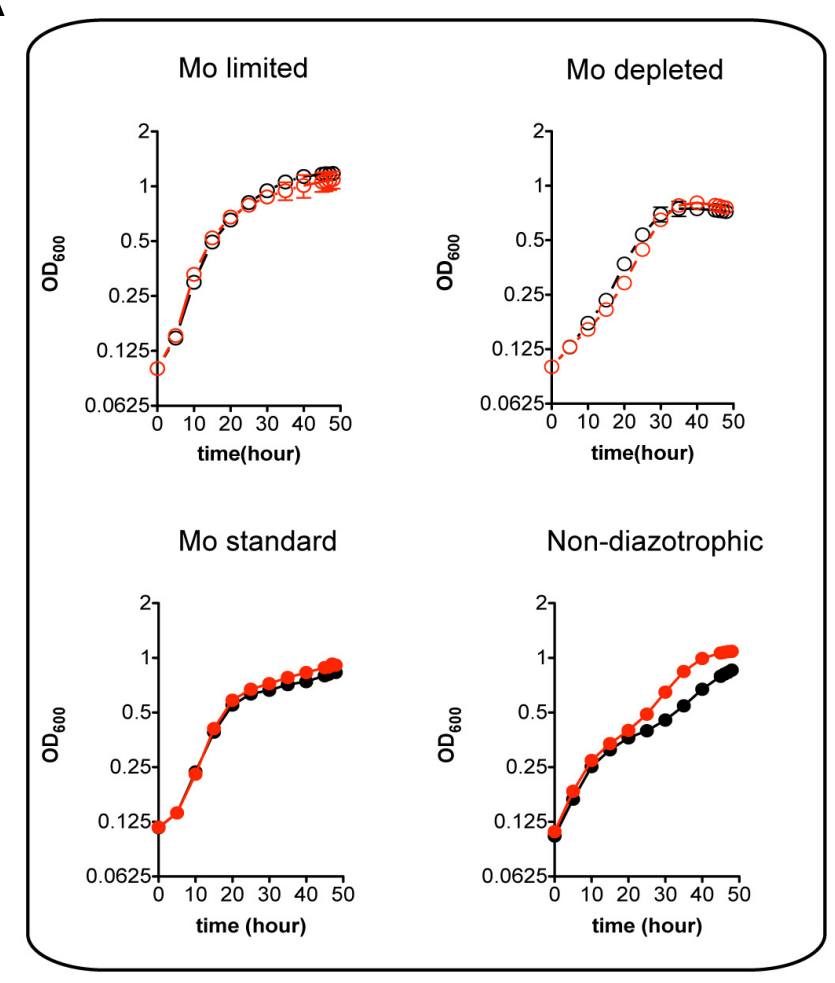

B

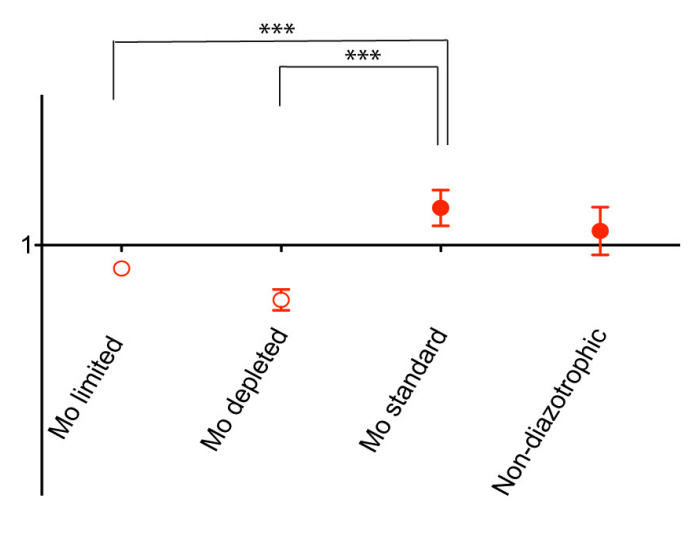

c
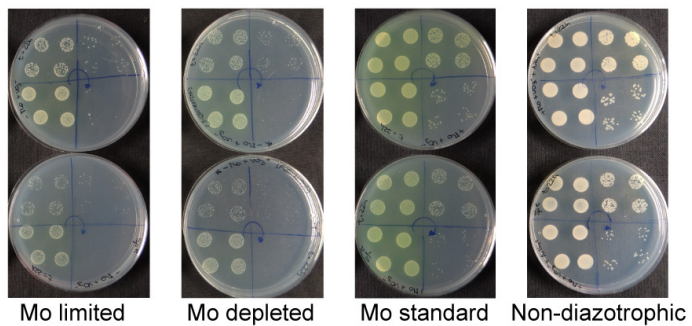

FIGURE 3 | Diazotrophic growth and $\mathrm{Cl}$ analysis of the MoSto mutant. (A) A. vinelandii DJ (wild type; black symbols) and UW394 ( $\triangle$ mosAB, red symbols) were cultured in Burk's modified N-free medium supplemented (close symbols) or not supplemented (open symbols) with $1 \mu \mathrm{M}$ molybdate. Mo-limited (4 nM), Mo-depleted and Mo standard $(1 \mu \mathrm{M})$ conditions were tested. All N-free media contained $1 \mu \mathrm{M}$ vanadate. Growth in Burk's modified medium containing ammonium acetate (non-diazotrophic) was carried out as control. (B) Cl values were obtained from mixed cultures (1:1 inoculum ratio) of DJ and UW394 strains. Data represent the mean and standard deviation of three or four independent experiments. ${ }^{* * *}$ Indicates unpaired $t$-test $P$-value $<0.001$. (C) Plates showing growth on solid media of mixed cultures that were pre-grown in liquid medium for $22 \mathrm{~h}$. Four $20-\mu \mathrm{l}$ drops with different dilutions $\left(10^{3}, 10^{4}, 10^{5}\right.$, and $\left.10^{6}\right)$ of the mixed culture were plated on each quarter of the plate. Arrows show the direction of the dilutions. Upper plates contain media lacking spectinomycin (to allow DJ and UW394 growth) whereas lower plates contain media supplemented with spectinomycin (to only allow UW394 growth).

activity open circles of Figures $\mathbf{2 A , B}$ ). This indicates that metal accumulated at MoSto is readily accessible for FeMo-co synthesis allowing maximum nitrogenase levels under transient Mo-limiting conditions. ( $v$ ) Prolonged Mo starvation equally affected Mo content and nitrogenase activity in wild type and MoSto mutant; (vi) 30\% nitrogenase activity remained in both strains after prolonged Mo starvation (Figure 2A) suggesting V-nitrogenase was being expressed.

Despite its pronounced impairment in Mo accumulation and nitrogenase activity, the MoSto mutant was only mildly affected in diazotrophic growth in Mo-limited conditions. This is consistent with the Mo requirements for maximum nitrogenase activity being much lower than the capacity of A. vinelandii to scavenge and accumulate Mo (Shah et al., 1984). In contrast, the MoSto mutant showed slightly better growth than wild type in the presence of $1 \mu \mathrm{M}$ molybdate both under diazotrophic and non-diazotrophic conditions (Figure 3A). Competitive index (CI) assays were carried out to analyze the $\triangle m o s A B$ phenotype in situations of competition for limiting Mo that are of environmental importance. In these assays growth interference takes place between the wild type and mutant strains. A CI $>1$ indicates that the mutant is more competitive than wild type whereas a $\mathrm{CI}<1$ indicates the mutant is less competitive. Figures 3B,C shows that the MoSto mutant is less competitive than wild type in diazotrophic growth under Mo starvation. CI was lowest under severe Mo starvation imposed by continuous growing in Mo-depleted medium consistent with a MoSto role as Mo reservoir. Interestingly the MoSto mutant was more competitive than wild type in the presence of $1 \mu \mathrm{M}$ molybdate in the medium. Because MoSto is present at similar levels in both conditions (see below) this result suggests that the energy burden of loading Mo into MoSto is not negligible.

\section{MoSto Protects $A$. vinelandii Nitrogenase From W Toxicity}

$\mathrm{W}$ is a well-known competitive inhibitor of Mo functions having negative effect on $A$. vinelandii growth (Keeler and Varner, 1957; Shah et al., 1984). Importantly, binding of W to MoSto has been shown to occur in vivo (Pienkos and Brill, 1981) and in vitro (Schemberg et al., 2007). The possible role of MoSto in protection against $\mathrm{W}$ toxicity was investigated by comparing $\triangle m o s A B$ diazotrophic growth to that of the 


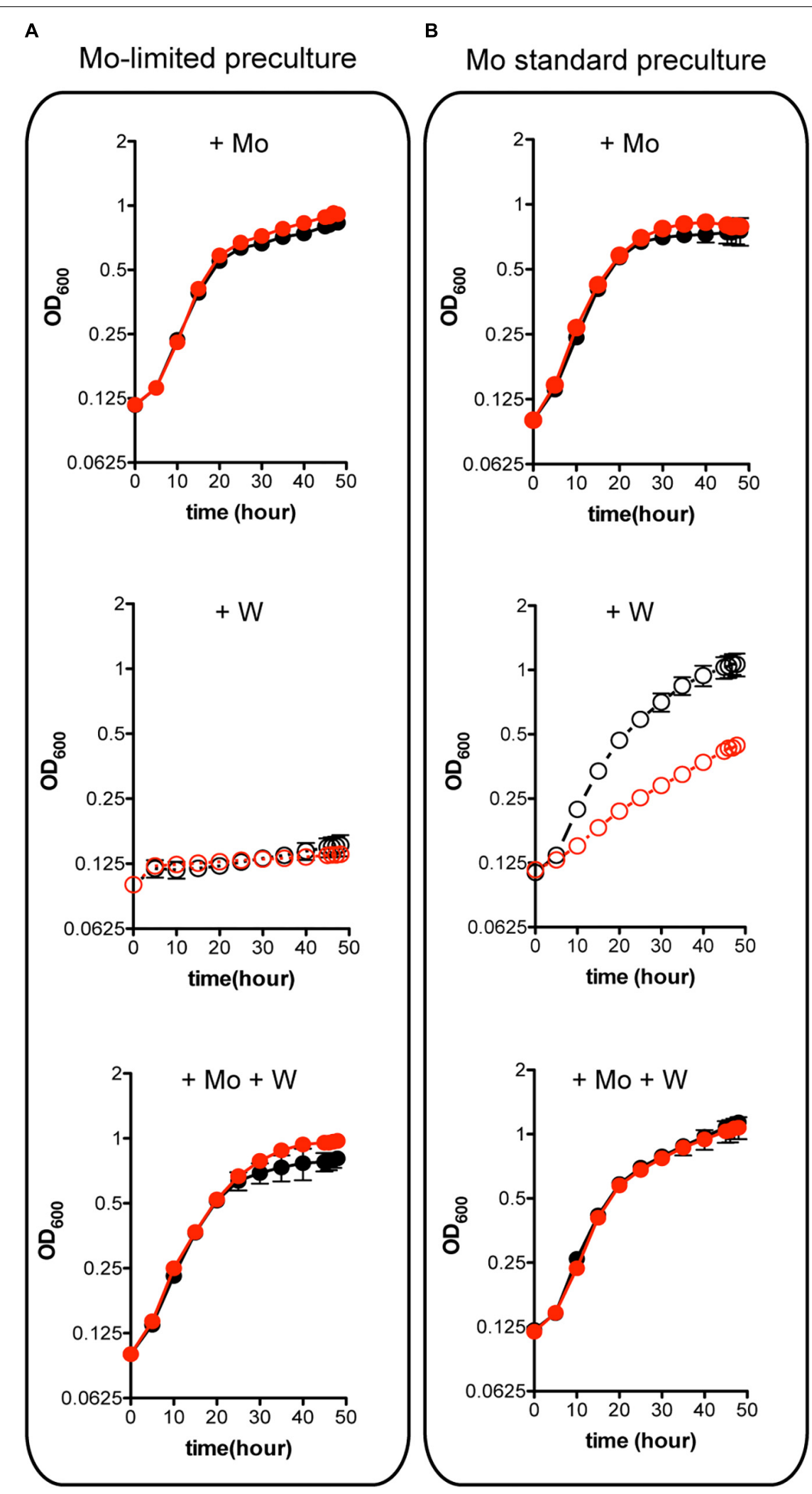

FIGURE 4 | Effect of $W$ on the diazotrophic growth of an $A$. vinelandii $\triangle$ mosAB strain. Mo-starved (A) or Mo standard (B) precultures of $A$. vinelandii DJ (wild type; black symbols) or UW394 ( $\triangle$ mosAB, red symbols) were inoculated into Burk's modified $\mathrm{N}$-free medium containing $1 \mu \mathrm{M}$ molybdate (close symbols) or 4 nM molybdate (open symbols). One $\mu \mathrm{M}$ tungstate was included when indicated and all media contained $1 \mu \mathrm{M}$ vanadate. Data represent mean \pm standard deviation of three independent experiments. 


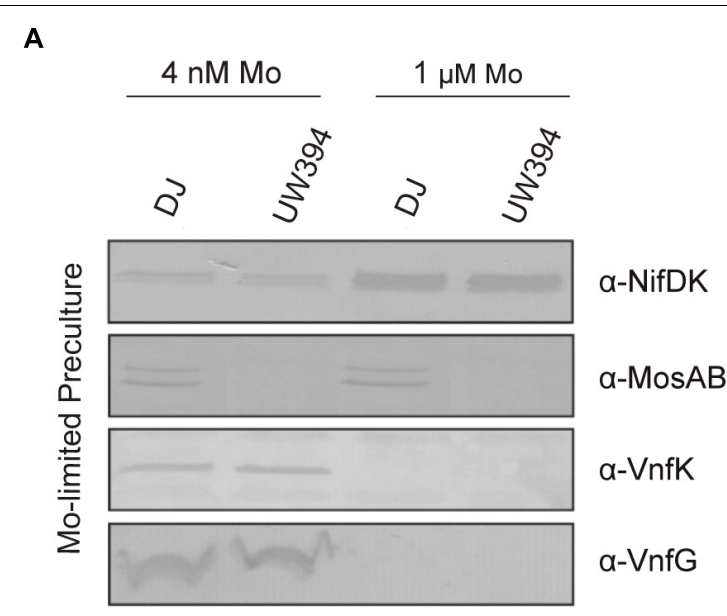

B

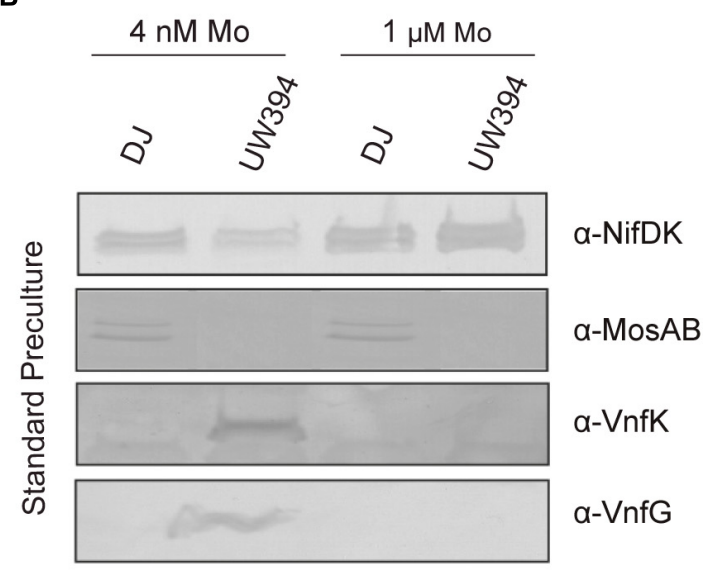

C

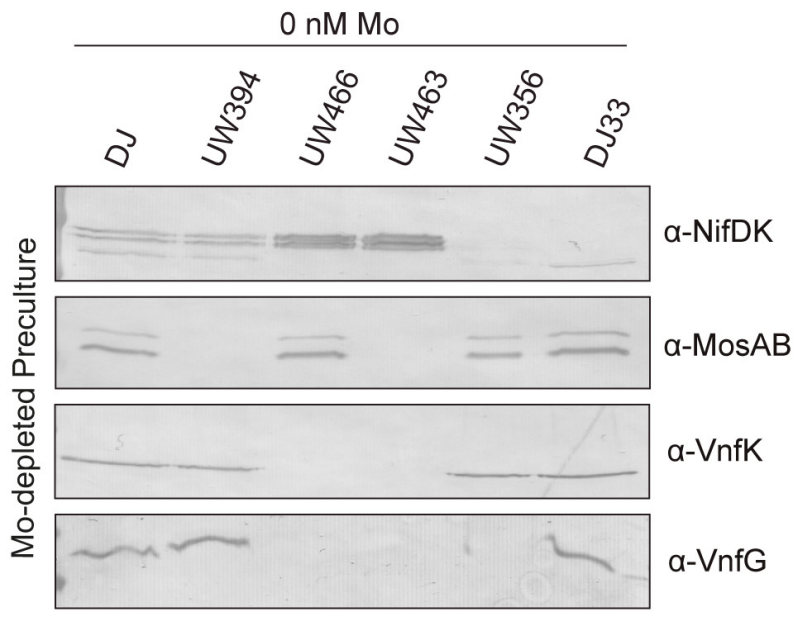

FIGURE 5 | Detection of Mo-nitrogenase and V-nitrogenase structural polypeptides in $A$. vinelandii. Mo-starved $(\mathbf{A}, \mathbf{C})$ or Mo standard (B) precultures of $A$. vinelandii DJ (wild type), UW394 ( $\triangle$ mosAB), UW466 ( $\Delta v n f \Delta a n f)$, UW463 ( $\Delta$ vnf $\triangle$ anf $\triangle$ mosAB), UW356 ( $\Delta$ nifA), or DJ33 ( $\Delta$ nifDK) were inoculated into Burk's modified $\mathrm{N}$-free medium containing either $\mathrm{O}, 4 \mathrm{nM}$ or $1 \mu \mathrm{M}$ molybdate to allow nitrogenase derepression. NifDK, VnfK, VnfG, and MosAB polypeptides accumulated in the cells were detected by Western blot analysis against specific antibodies. wild type at environmentally relevant metal concentrations (Figure 4). Mo starved precultures of both wild type and MoSto mutant were highly sensitive to inhibition by $1 \mu \mathrm{M}$ W (a W/Mo ratio of 250) (Figure 4A) an amount that could exceed the W trapping capacity of MoSto. In contrast, when cells grown in Mo standard conditions were transferred to Molimited conditions in the presence of $\mathrm{W}$ the wild type strain grew normally while the MoSto mutant was clearly inhibited (Figure 4B). The differential behavior of wild type and the $\triangle m o s A B$ mutant under transient Mo starving conditions can be rationalized considering MoSto as reservoir that continuously provides Mo for FeMo-co synthesis thus avoiding $\mathrm{W}$ toxicity. In all cases, co-presence of $1 \mu \mathrm{M}$ molybdate in the medium protected from $1 \mu \mathrm{M}$ tungstate inhibition whereas $1 \mu \mathrm{M}$ vanadate did not.

\section{Deletion of mosAB Genes Affects V-Nitrogenase Accumulation Under Mo Deficiency}

Mo tightly represses expression of V-nitrogenase (Bishop and Joerger, 1990). However, V-nitrogenase and Mo-nitrogenase transcripts coexist when molybdate levels in the medium are in the range of 10-50 nM (Jacobson et al., 1986). Because the presence or absence of MoSto largely determines intracellular Mo concentration, the effect of $\triangle \operatorname{mos} A B$ mutation on the accumulation of Mo-nitrogenase and V-nitrogenase structural components in relation to changes in Mo availability was investigated. On one hand, Mo starved cells were transferred to the same Mo-limiting conditions (4 $\mathrm{nM} \mathrm{Mo)} \mathrm{or} \mathrm{to}$ medium with standard molybdate $(1 \mu \mathrm{M}$ Mo). Standard medium allowed maximum NifDK accumulation and repressed VnfDGK synthesis, as expected (Figure 5A). Under Molimiting conditions, Nif polypeptides were present at much lower levels and coexisted with Vnf polypeptides, both in the wild type and in the $\triangle \operatorname{mos} A B$ strain. On the other hand, transferring Mo sufficient cells to Mo-limited medium (Mo step down) readily derepressed $v n f D G K$ expression in the $\triangle \operatorname{mos} A B$ strain but not in the wild type, which in contrast accumulated more NifDK than the mutant (Figure 5B). Importantly, MoSto was accumulated to similar levels under all Mo concentrations tested in this study, in agreement with previous observations (Pienkos and Brill, 1981; Fenske et al., 2005). These results indicates that the buffering effect that MoSto has in Mo homeostasis (Figure 2B) softens the regulatory response to transient Mo limitation repressing early $v n f$ expression and maintaining higher Nif-dependent nitrogenase activity.

\section{The mosAB Deletion Has No Effect on the Activity of Alternative Nitrogenases}

The $\triangle \operatorname{mos} A B$ mutation was also introduced into a $\triangle v n f D G K$ $\triangle a n f D G K$ strain, lacking the $\mathrm{V}$ and Fe-only nitrogenase structural components, to investigate a possible role in these alternative systems. Cells cultured in Mo-depleted medium were derepressed for nitrogenase in either Mo-depleted or Mostandard media supplemented with $1 \mu \mathrm{M}$ vanadate. In vivo 
TABLE 2 | Ratio of ethane to ethylene production in MoSto deficient strains derepressed in media with different metal availability.

\begin{tabular}{|c|c|c|c|}
\hline Strain ${ }^{a}$ & Metal & $\mathrm{C}_{2} \mathrm{H}_{4}^{\mathrm{b}}$ & $\mathrm{C}_{2} \mathrm{H}_{6} / \mathrm{C}_{2} \mathrm{H}_{4}(\%)^{\mathrm{c}}$ \\
\hline \multirow[t]{2}{*}{ DJ } & V & $1.58 \pm 0.07$ & 0.63 \\
\hline & Mo, V & $13.65 \pm 0.55$ & 0 \\
\hline \multirow[t]{2}{*}{ UW394 } & V & $1.57 \pm 0.01$ & 0.71 \\
\hline & Mo, V & $15.24 \pm 1.04$ & 0 \\
\hline \multirow[t]{2}{*}{ UW466 } & V & $1.83 \pm 0.28$ & 0.65 \\
\hline & Mo, V & $13.94 \pm 1.03$ & 0 \\
\hline \multirow[t]{2}{*}{ UW463 } & V & $1.92 \pm 0.18$ & 0.60 \\
\hline & Mo, V & $14.95 \pm 1.04$ & 0 \\
\hline \multirow[t]{2}{*}{ UW356 } & V & $0.47 \pm 0.01$ & 1.00 \\
\hline & Mo, V & 0 & 0 \\
\hline \multirow[t]{2}{*}{ DJ33 } & V & $1.64 \pm 0.59$ & 1.22 \\
\hline & Mo, V & 0 & 0 \\
\hline
\end{tabular}

aprecultures grown in $\mathrm{NH}_{4}^{+}$-containing Mo-depleted medium were transferred to $\mathrm{N}$-free medium supplemented with $1 \mu \mathrm{M}$ of the indicated metal. ${ }^{b}$ Activities are in $\mathrm{nmol}$ ethylene per min per $\mathrm{ml}$ at an $O D_{600}$ of 1 . Data represent mean and standard deviations of three independent experiments. ${ }^{C} \mathrm{C}_{2} \mathrm{H}_{6}$ production shown as $\%$ of $\mathrm{C}_{2} \mathrm{H}_{4}$.

nitrogenase activities were estimated by measuring acetylene reduction into ethylene and ethane (Table 2), an exclusive property of alternative nitrogenases (Dilworth et al., 1987) while the presence of Mo- or V-nitrogenase structural components under Mo-depleted conditions was determined by immunoblot analysis (Figure 5C). Importantly, all Mo-depleted strains contained less than $0.7 \mathrm{pmol}$ Mo per ml of culture at $\mathrm{OD}_{600}$ of 1 at $8 \mathrm{~h}$ of derepression.

Strains with intact Mo-nitrogenase structural genes produced maximum levels of ethylene and repressed alternative nitrogenase structural proteins in media containing $1 \mu \mathrm{M}$ Mo and $1 \mu \mathrm{M} \mathrm{V}$, as expected. Under Mo-depleted conditions, the wild type strain as well as DJ33 ( $\Delta$ nifDK) and UW356 ( $\triangle$ nifA, encoding the nif gene transcriptional activator) exhibited concomitant production of ethylene and ethane diagnostic of alternative nitrogenase activity. Surprisingly, the $\triangle$ vnfDGK $\triangle a n f D G K$ strains UW466 and UW463 exhibited similar ethylene and ethane production levels than $\mathrm{vnf}^{+} \mathrm{anf}^{+}$strains (Table 2) despite the absence of Vnf proteins (Figure 5C). However, these strains accumulated high levels of NifDK. This observation suggests the plausible insertion of FeV-co into NifDK under Mo-depleted conditions, which would be responsible for the observed residual nitrogenase activities. No effect of the $\operatorname{mos} A B$ mutation on nitrogenase activity was detected in this condition in any of the tested genetic backgrounds.

\section{DISCUSSION}

The A. vinelandii MoSto protein is rather unique both in function and in amino acid sequence having no known homologs in protein databases. It has the capacity to accumulate massive amounts of Mo (over 100 atoms per MoSto), which would be a favorable trait to add to engineered nitrogen fixing plants expressing bacterial Mo-nitrogenase provided this Mo was directly available to nitrogenase FeMo-co biosynthesis. However, such a direct transfer had not yet been experimentally proven. Here we show that the Mo stored at MoSto is directly available for FeMo-co synthesis in vitro and that interaction of MoSto with FeMo-co biosynthetic proteins stimulates cofactor synthesis. The exact mechanism by which this interaction stimulates cofactor synthesis is unclear. Direct transfer of Mo from NifQ to the NifEN/NifH complex during FeMo-co biosyntesis in vitro has been reported (Hernandez et al., 2008). The similarities between the Mo-Fe-S clusters found in NifQ and in NifEN purified from a $\Delta$ nifH background (Soboh et al., 2006; George et al., 2007) support such direct connection. The expected primary path for molybdenum incorporation into FeMo-co would include molybdate transport, storage at MoSto, transfer to NifQ, and finally to the NifEN/NifH complex. However the results shown here indicate some degree of NifQ function replacement by MoSto in the in vitro system. Mo release from MoSto occurs spontaneously in vitro above $\mathrm{pH} 6.8$ (Schemberg et al., 2008) and perhaps an interaction with NifEN

TABLE 3 | Bacterial strains and plasmids.

\begin{tabular}{lll}
\hline Strain or plasmid & Genotype & Resource \\
\hline $\begin{array}{l}\text { Escherichia } \\
\text { coli }\end{array}$ & & \\
$\mathrm{DH} 5 \alpha$ & $\mathrm{F}-\varphi 80 \Delta \mathrm{lacZM15} \Delta$ (lacZYA- $^{-}$ & Sambrook and \\
& $\begin{array}{l}\text { argF)U169 deoP recA1 endA1 } \\
\left.\text { hsdR17 (rK } \mathrm{mK}^{-}\right)\end{array}$ & Russell, 2001 \\
BL21(DE3)pLysS & $\mathrm{F}^{\prime}-$ ompT galldcm][lon] hsdsB (rB ${ }^{-}$ & Studier and Moffatt, \\
& $\mathrm{mB}^{-} ;$an $E$. coli B strain) with DE3 & 1986 \\
& and pLysS
\end{tabular}

Azotobacter vinelandii

DJ

UW394

UW466

UW463 and pLysS

UW356

Highly transformable variant of $\mathrm{OP}$

Setubal et al., 2009 $\triangle m o s B A:: s p c$

$\Delta v n f D G K::$ tet $\rightarrow ; \Delta$ anfDGK::kan $\rightarrow$

$\triangle$ mosBA::spc; $\triangle$ vnfDGK::tet $\rightarrow$; $\triangle a n f D G K:: k a n \rightarrow$

DJ33

$\triangle$ nifA::spc

$\Delta$ nifDK

\section{Plasmids}

pBluescript KS (+) Cloning vector

pGEMT-vector Cloning vector

pET28a (+)

Expression vector

This study

This study

This study

Poza-Carrion et al., 2014

Robinson et al., 1986 pUC4K

Vector containing Kan resistance cassette

pBBR1-MCS3

Vector containing Tet resistance cassette

$\mathrm{pHP} 45 \Omega$

Vector containing Spc/Sm resistance cassette

Agilent

Promega

Novagen

Pharmacia

Obranic et al., 2013

Prentki and Krisch, 1984

pRHB268

pGEMT carrying mosBA flanking regions and spc cassette

pN2MN14 pBSKS(+) carrying anfDGK flanking regions and kan cassette

pN2MN18 pBSKS(+) carrying vnfDGK flanking regions and tet cassette

pN2MN72 mosBA genes cloned into pET28a (+) This study 
facilitated Mo transfer. In this context, it is interesting to note that a NifH-independent pathway for Mo transfer into NifEN was previously postulated (Soboh et al., 2006). Thus, the ability of MoSto to donate Mo for FeMo-co synthesis might also be relevant in vivo and could underlie the phenotypic reversal of $n i f Q^{-}$by excess molybdate (Joerger and Bishop, 1988; Rodriguez-Quinones et al., 1993).

Mo release from MoSto and its utilization for in vitro for FeMo-co synthesis occurs at $\mathrm{pH} 7.5$ in the presence of $1.23 \mathrm{mM}$ ATP and an ATP regenerating enzyme in the reaction mixtures. It is known that MoSto catalyzed ATPhydrolysis promotes formation of polyoxomolybdate clusters inside the MoSto cage while molybdate release from MoSto is favored at $\mathrm{pH}$ 7.5, but only after ATP is consumed (Poppe et al., 2018). Therefore, the buffer composition of the in vitro FeMo-co synthesis assay should preclude Mo release from MoSto. This discrepancy can be explained if FeMo-co biosynthesis and nitrogenase reconstitution alter the equilibrium of Mo binding/release by removing Mo from the available pool.

MoSto endows $A$. vinelandii with the ability to maintain high Mo-dependent diazotrophic growth rates under transient Mo limitation thus increasing strain competitiveness. It also confers certain degree of protection against W, a Mo antagonist that renders inactive molybdoenzymes. Normally $W$ toxicity for nitrogen-fixing cells of $A$. vinelandii is evident when large excess of $\mathrm{W}$ over Mo (i.e., for W/Mo ratios > 150) is present in the culture medium (Keeler and Varner, 1957). This is not the case of the MoSto mutant, which exhibits high sensitivity to equimolar concentrations of $\mathrm{M}$ and W. Similar W sensitive phenotypes have been observed in mutants deficient in catechol siderophore production (Wichard et al., 2008). Both, Mo fluctuating conditions, including severe Mo limitation, and the presence of $\mathrm{W}$ at concentrations equal or higher than Mo, are environmentally relevant conditions. The average concentration of molybdate in terrestrial environments is $50 \mathrm{nM}$ but its distribution is irregular (Hernandez et al., 2009). The advantage that MoSto might confer in the environment is however obscured under laboratory growth conditions in which large excess of molybdate is present in the medium.

Under Mo sufficient conditions more than 95\% intracellular Mo is bound to MoSto. However, Mo-storage is not essential to achieve maximum nitrogenase activity as long as the growth medium contains excess molybdate (Figure 2B). This indicates that $A$. vinelandii ATP-dependent high affinity molybdate transport (Mouncey et al., 1995) is independent of the storage process and that molybdate uptake rates are enough to support maximum nitrogenase activity. Mo loading into MoSto is also an ATP-dependent process (Allen et al., 1999; Schemberg et al., 2008), which might impose an energy burden to the cell. This fact would explain why the mutant strain lacking MoSto is more competitive than the wild type under non-diazotrophic Mo-sufficient growth conditions. There are discrepancies in the literature as to whether or not MoSto expression is regulated by Mo. Pienkos reported constitutive MoSto expression (Pienkos and Brill, 1981) while Fenske found MoSto in cells grown at molybdate concentrations as low as $1 \mathrm{nM}$ but not in Mo-free medium (Fenske et al., 2005). Our results are in line with constitutive expression since we were able to detect MoSto in cells grown in medium with Mo levels below an ICP-MS detection limit of $0.05 \mathrm{ppb}$. It is however possible that molybdate traces below our experimental detection limit are enough to induce MoSto expression.

We observed the co-existence of NifDK and VnfDGK polypeptides in cells grown under severe Mo-limiting or Modepleted conditions, although NifDK levels were much lower than those at standard Mo conditions (Figure 5). Transcripts of nif and $v n f$ structural genes had been shown to co-exist at concentrations of Mo in the medium between 10 and $50 \mathrm{nM}$ (Jacobson et al., 1986) but neither at $4 \mathrm{nM}$ nor in Mo-depleted medium. It is likely that these discrepancies are due to the different sensitivity of Mo determination or product (either RNA or antigen) detection methods. Under Mo-deplete conditions the $\Delta v n f \Delta a n f$ mutants, lacking VnfDGK, appear to compensate with higher amounts of NifDK. It is know that the alternative nitrogenases can catalyze the reduction of acetylene by either two or four electrons to yield ethylene and ethane, respectively (Dilworth et al., 1988). Surprisingly, all strains exhibited activity with features typical of alternative nitrogenases under Modepleted conditions regardless of the presence of VnfDGK. Thus, simultaneous contributions from both systems cannot be ruled out. Hybrid Mo-nitrogenases carrying the cofactor of the V-nitrogenase (Moore et al., 1994) or even a biosynthetic precursor to FeMo-co (Soboh et al., 2010) have been generated in vitro and were shown to exhibit altered substrate specificities and product formation. Although in standard derepressing conditions the NafY protein seems to have a discriminating role in the insertion of Mo-nitrogenase active site metal cofactor (Rubio et al., 2004) the situation under extremely Mo-deficient conditions had not been tested.

In the absence of MoSto Vnf polypeptides are readily derepressed upon Mo step down, in contrast to the wild type that requires prolonged Mo starvation to initiate derepression (Figure 5B). Thus, the buffering effect of MoSto may also be important to maintain tight regulation of nitrogenase with different metal specificities.

\section{CONCLUSION}

Under transient Mo-limiting conditions MoSto mutants showed low Mo accumulation levels, lost the ability to repress expression of the V-dependent nitrogenase, exhibited high sensitivity to $\mathrm{W}$ inhibition, and were less competitive than wild type in diazotrophic growth. Importantly, the in vitro FeMo-co synthesis assay establishes the donation of Mo from MoSto to FeMo-co biosynthetic proteins via direct interaction. MoSto provides robust Mo-dependent nitrogen fixation under Molimiting conditions to its prokaryotic host. A corollary to these results is the need to incorporate the MoSto genes into the prokaryotic gene complement required to engineer nitrogen-fixing plants (Allen et al., 2017; Buren et al., 2017; Buren and Rubio, 2018). 


\section{MATERIALS AND METHODS}

\section{Generation of $A$. vinelandii Strains}

Strains and plasmids used are listed in Table 3. A. vinelandii DJ (wild type) (Setubal et al., 2009), UW356 ( $\Delta$ nifA::spc) (Poza-Carrion et al., 2014) and DJ33 ( $\Delta$ nifDK) (Robinson et al., 1986) have been previously described. Strains UW394, UW466, and UW463 carrying in-frame deletions of the $\operatorname{mos} A B$, vnfDGK/anfDGK, and mosAB/vnfDGK/anfDGK genes, respectively, were generated in this work. Deletions were incorporated into the $A$. vinelandii chromosome by transformation and gene replacement as described (Dos Santos, 2011).

Strain UW394 was generated by transforming $A$. vinelandii DJ with plasmid pRHB268 in which the mosBA genes had been replaced by an spectinomycin resistance cassette

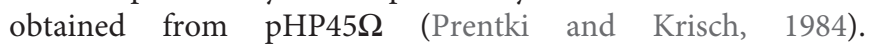
pRHB268 is a derivative of pRHB266, which contains the $m o s B A$ region amplified by $\mathrm{PCR}$ using oligonucleotides $5^{\prime}$-CGCTCGCCCAGCTCGGTCAGGCGCA- $3^{\prime}$ and 5'-CAG AGACCTGCTCGCCAGCTGAAATCC- $3^{\prime}$ and cloned into pGEM-T. pRHB266 was digested with BspEI/AgeI restriction enzymes to eliminate the mos $B A$ genes, followed by Klenow treatment and blunt end ligation for the insertion of the SmaI-digested spectinomycin resistance cassette.

In-frame deletions of alternative nitrogenases were generated by co-introducing plasmids pN2MN14 and pN2MN18 into $A$. vinelandii DJ and UW394 to generate strains UW466 and UW463, respectively. To generate $\mathrm{pN} 2 \mathrm{MN} 14, \mathrm{DNA}$ regions flanking anfDGK were amplified by PCR using oligonucleotides $5^{\prime}$ GGTTTCTCGAGATGACTCGTAAAGTAGCCAT- $3^{\prime}$ and $5^{\prime}$-GA TGGGATCCGACACATCTCCTTTAGAGTGA-3' for the region upstream anfD and oligonucleotides 5'-ACCTGGATCCG

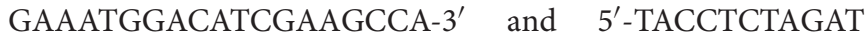
GAGGACCCATTCCTTGTTC-3' for the region downstream anfK. PCR products were digested with XhoI/BamHI/XbaI and cloned into XhoI and XbaI sites of pBlueScript KS (+) in a quadruple ligation reaction together with BamHI-digested kanamycin resistance cassette obtained from plasmid pUC4K by amplifying a PCR product using oligonucleotides $5^{\prime}$ AATTGGATCCGGGAAAGCCACGTTGTGTCTC- $3^{\prime}$ and $5^{\prime}$ -AATTGGATCCCTTTTGCTTTGCCACGGAACGG-3'. To generate pN2MN18, DNA regions flanking $v n f D G K$ were amplified by PCR using oligonucleotides $5^{\prime}$-AGGC CTCGAGTGCATGACCGATGGGAC- $3^{\prime}$ and $5^{\prime}$-CCATGGA TCCGATTGAAGTCTCCTCGGCTCT-3' for the region upstream $v n f D$ and oligonucleotides $5^{\prime}$-GTGGTGGATC CAGGTGCCGGAGCGGTTTCC ${ }^{\prime}-3^{\prime}$ and 5'-GGGTTCTA GAAGTCCAGGCGGACATGGC-3' for the region downstream vnfK. PCR products were digested with XhoI/BamHI/XbaI and cloned into XhoI and XbaI sites of pBluescript KS $(+)$ in a quadruple ligation reaction together with BamHIdigested tetracycline resistance cassette obtained from plasmid pBBR1-MCS3 by amplifying a PCR product using oligonucleotides $\quad 5^{\prime}$-CCGGGATCCCTCATGTTTGACAGCTT
ATCAT- $3^{\prime}$ and $5^{\prime}$-CCGGGATCCGGAGTGGTGAATCCGTTA GC-3' (Obranic et al., 2013).

Isolation of genomic DNA from A. vinelandii strains was performed by using DNAeasy ${ }^{\text {TM }}$ Tissue Kits (Qiagen). Generated A. vinelandii mutant strains were confirmed by PCR analysis and by immunoblot analysis with appropriate antibodies.

Escherichia coli DH5 $\alpha$ was used for cloning procedures. Plasmid constructions, PCR DNA amplifications, and E. coli transformations were carried out by standard methods (Sambrook and Russell, 2001). Restriction analysis and DNA sequencing was used to confirm accuracy of all DNA constructs. To overexpress the $A$. vinelandii $m o s B A$ genes in $E$. coli, the mosBA genomic region of A. vinelandii was amplified by PCR using oligonucleotides $5^{\prime}$-GCGCGAATTCGCCAACTCGACAGCG-3' and $5^{\prime}$-GCGC GCGGCCGCTCAGGCCGGACGCACA-3', digested with EcoRI and NotI, and cloned into the EcoRI and Not I restriction sites of expression vector pET28a (+) to generate plasmid pN2MN72.

\section{Bacterial Strains and Growth Conditions}

Escherichia coli $\mathrm{DH} 5 \alpha$ was cultivated in Luria-Bertani medium at $37^{\circ} \mathrm{C}$ with shaking (250 r.p.m.). Antibiotics were added at standard concentrations (Sambrook and Russell, 2001). For MoSto overexpression experiments, E. coli BL21(DE3) pLysS strain was transformed with plasmid pN2MN72 and cultivated in $4 \mathrm{~L}$ fermentors in Luria-Bertani (LB) medium supplemented with $0.3 \mathrm{mM}$ ammonium ferric citrate, $0.3 \mathrm{mM}$ cysteine and, when indicated, $1 \mathrm{mM} \mathrm{Na} 2 \mathrm{MoO}_{4}$. Fermentor cultures started at a cell $\mathrm{OD}_{600}$ of 0.022 and proceeded for $18 \mathrm{~h}$ at $30^{\circ} \mathrm{C}$ with air sparging (2.5 l/min) and stirring (300 r.p.m.).

Azotobacter vinelandii strains were cultivated in Burk's modified medium (containing $28 \mathrm{mM}$ ammonium acetate) or in Burk's modified N-free medium at $30^{\circ} \mathrm{C}$ (Strandberg and Wilson, 1968) with modifications of metal contents (Mo, Fe, $\mathrm{V}$, and $\mathrm{W}$ ), when indicated. Antibiotics were added at standard concentrations (Curatti et al., 2005). Regarding Mo, three types of culture medium were used here and are defined as Molimited, Mo-depleted and Mo-standard. Mo-limited medium was prepared without molybdate and contained chemical components of high purity. All glassware used was acid washed and rinsed with milliQ water (Chatterjee et al., 1994). Mo-limited medium contained 2.1-4.4 nM Mo as determined by ICP-MS. Depletion of Mo traces still remaining in Mo-limited medium was achieved by incubating Mo-starved A. vinelandii DJ cells in $4 \mathrm{~L}$ of Burk's modified N-free Mo-limited medium supplemented with $1 \mu \mathrm{M} \mathrm{NaVO}$. After $1 \mathrm{~h}$ of incubation, $2 \mathrm{~L}$ were collected and cells removed by centrifugation followed by filtration of the supernatant. The remaining $2 \mathrm{~L}$ of culture were supplemented with $28 \mathrm{mM}$ ammonium acetate, incubated for 1 additional hour, after which cells were removed by centrifugation followed by filtration of the supernatant. Polypropylene plastic was used to harvest Mo-depleted medium. Collected medium remained sterile for months on the shelf. Mo levels in Mo-depleted medium were below the detection limit of the ICP-MS. Mo-depleted medium was used for competitive index analysis and the analysis of $\mathrm{Anf}^{-} \mathrm{Vnf}^{-}$strains. Mo-standard medium contained $1 \mu \mathrm{M}$ $\mathrm{Na}_{2} \mathrm{MoO}_{4}$ and was prepared by standard procedures. 
For nitrogenase derepression experiments, inoculum cultures were previously grown in Mo-limited Burk's modified medium (Mo-starving conditions), in Mo-depleted Burk's modified medium (Mo-depleted conditions), or in Burk's modified medium (Standard conditions). Mo-starved inoculum cultures had been previously grown and transferred at least three times in Mo-limited Burk's modified medium. A. vinelandii cells were then collected by centrifugation, washed with $\mathrm{N}$-free Mo-limited medium and resuspended in $\mathrm{N}$-free medium supplemented with $1 \mu \mathrm{M} \mathrm{NaVO}$ and lacking or containing $\mathrm{Mo}$, as indicated in each experiment. Similar procedure using Mo-depleted medium was followed to obtain Modepleted inoculum cultures. Each derepression culture was inoculated at an optical density at $600 \mathrm{~nm}\left(\mathrm{OD}_{600}\right)$ of 0.3 and further cultivated for at least $4 \mathrm{~h}(8 \mathrm{~h}$ for anf $v n f$ mutants) at $30^{\circ} \mathrm{C}$ with shaking (200 r.p.m.). At different times during derepression, culture samples were collected and subjected to the following analyses: determination of in vivo acetylene reduction activity, determination of cellular-bound molybdenum, growth as estimated by $\mathrm{OD}_{600}$, and detection of MoSto, NifDK, and VnfDGK proteins y immunoblot. Polypropylene filter tips were used for sample manipulation to prevent molybdenum traces.

For growth curves, A. vinelandii strains were cultured in $\mathrm{NH}_{4}^{+}$-containing (nitrogenase repressing) solid Burk's modified medium under Mo-limited or Standard conditions for at least 3 days. When Mo-starved cells were required, A. vinelandii strains were inoculated into Mo-limited Burk's modified medium at least three times. Individual colonies were inoculated into liquid Burk's modified medium and cultures were grown overnight to an $\mathrm{OD}_{600} \approx 2$. Inoculum cultures were then used to inoculate liquid $\mathrm{N}$-free (nitrogenase derepressing) Burk's modified medium with different $\mathrm{Mo}, \mathrm{V}$, and $\mathrm{W}$ contents: $1 \mu \mathrm{M} \quad \mathrm{NaVO}_{3} ; 1 \mu \mathrm{M} \quad \mathrm{Na}_{2} \mathrm{MoO}_{4}$ plus $1 \mu \mathrm{M}$ $\mathrm{NaVO}_{3} ; 1 \mu \mathrm{M} \mathrm{NaVO}_{3}$ plus $1 \mu \mathrm{M} \mathrm{Na}_{2} \mathrm{WO}_{4}$; and $1 \mu \mathrm{M}$ $\mathrm{Na}_{2} \mathrm{MoO}_{4}$ plus $1 \mu \mathrm{M} \mathrm{NaVO}$ plus $1 \mu \mathrm{M} \mathrm{Na} \mathrm{WO}_{4}$. Addition of $\mathrm{NaVO}_{3}$ permitted expression of $\mathrm{V}$-nitrogenase under Molimited conditions. Non-diazotrophic growth conditions in Burk's modified medium were analyzed as controls. Three hundred $\mu \mathrm{l}$ cultures were incubated at $30^{\circ} \mathrm{C}$ in 96 -well plates at intensive speed setting for $48 \mathrm{~h}$ in a Bioscreen $\mathrm{C}$ apparatus (Thermo Fisher).

\section{Competitive Index Assay}

Competitive index (CI) was defined as the mutant-to-wild-type ratio within the output sample, divided by the corresponding ratio in the inoculum (Macho et al., 2007). Precultures of wild type and UW394 were grown in Burk's modified medium to exponential phase, mixed to a final $\mathrm{OD}_{600}$ of 0.1 , and used to inoculate the Burk's modified $\mathrm{N}$-free medium as indicated in each experiment. Molybdenum and nitrogen regimes established in the precultures were maintained during cogrowth in liquid medium. Twenty $\mu \mathrm{l}$ of serial dilutions of the mixed cultures were sampled at incubation times 0 and $22 \mathrm{~h}$ and plated onto solid Burk's modified $\mathrm{N}$-free medium and solid Burk's modified N-free medium supplemented with spectinomycin (to inhibit growth of the wild type strain). Time
$0 \mathrm{~h}$ determinations give input mutant to wild type ratios whereas time $22 \mathrm{~h}$ determinations give output CI values. Calculated CIs are the mean of three independent experiments with standard errors.

\section{In vivo Nitrogenase Activity Assays}

In vivo nitrogenase activity was determined by the acetylene reduction assay (Stewart et al., 1967) with ethylene and ethane formation being analyzed in a Shimadzu GC-2014 gas chromatograph. Five $\mathrm{ml}$ culture samples were transferred to $26 \mathrm{ml}$ vials sealed with rubber stoppers. After injection of $1.5 \mathrm{ml}$ acetylene to each vial, reactions proceeded for $30 \mathrm{~min}$ at $30^{\circ} \mathrm{C}$ with shaking. Reactions were then stopped by addition of $0.1 \mathrm{ml}$ of $8 \mathrm{M} \mathrm{NaOH}$. To detect ethylene, $50 \mu \mathrm{l}$ samples of the gas phase of each reaction assay were injected in a PoraPak $\mathrm{N}$ 80-100 column. Gas chromatography temperature conditions were: $150^{\circ} \mathrm{C}$ at the injection port; $90^{\circ} \mathrm{C}$ at the column and $\mathrm{N}_{2}$ carrier gas; and $150^{\circ} \mathrm{C}$ at the flame ionization detector (FID). To detect ethylene and ethane, $500 \mu \mathrm{l}$ of the gas phase of each reaction assay were injected in a PoraPak N 50-80 column. Gas chromatography temperature conditions were: $180^{\circ} \mathrm{C}$ at the injection port; $60^{\circ} \mathrm{C}$ at the column and $\mathrm{N}_{2}$ carrier gas; and $200^{\circ} \mathrm{C}$ at the FID.

\section{Mo Determinations}

Determination of Mo content in Mo-limited and Mo-depleted media was carried out by ICP-MS. Mo content of $A$. vinelandii cells was determined by Inductively Coupled Plasma Optical Emission Spectroscopy (ICP-OES) or by Inductively Coupled Plasma Mass Spectroscopy (ICP-MS). Cell culture samples $(40 \mathrm{ml})$ were harvested by centrifugation at 5,000 r.p.m. for $5 \mathrm{~min}$ at $4^{\circ} \mathrm{C}$, washed three times with Mo-limited or Modepleted medium, and collected under the same conditions. Pellets were dried at $100^{\circ} \mathrm{C}$ until ashes were formed and then resuspended in 5\% nitric acid solution for ICP analysis. Culture samples were analyzed by ICP-OES at the ionomic service of the CEBAS-CSIC (Spain) or at ICP-MS at the Unit of Metal Analysis of the University of Barcelona Scientific and Technology Center (Spain) if Mo levels were too low to be detected by ICP-OES. Whole cell Mo contents are referred to as pmol Mo per cells contained in $1 \mathrm{ml}$ of culture at an $\mathrm{OD}_{600}$ equal to 1 $\left(2.2 \times 10^{8}\right.$ cells $)$.

Mo determinations in purified MoSto preparations were carried out by ICP-OES or ICP-MS. The colorimetric method of Cárdenas (Cárdenas and Mortenson, 1974) was used to follow Mo-containing fractions during purification of MoSto from A. vinelandii cells.

\section{Protein Methods}

Protein concentration was determined by the bicinchoninic acid method with BSA as the standard (Smith et al., 1985). Procedures for SDS-PAGE (Laemmli, 1970) and immunoblot analysis (Brandner et al., 1989) have been described. Protein samples for immunoblot analyses were prepared by mixing pelleted cells with $100 \mathrm{mM}$ Tris- $\mathrm{HCl}$ at a final $\mathrm{OD}_{600}$ of 4 , adding Laemmli buffer $4 \mathrm{X}$ supplemented with $0.1 \mathrm{M}$ DTT, heating at $95^{\circ} \mathrm{C}$ for $3 \mathrm{~min}$, and removing debris by centrifugation at 12000 
r.p.m. for $2 \mathrm{~min}$ to obtain solubilized protein samples. Six $\mu 1$ samples were loaded per lane for NifDK and MosAB detection, $8 \mu \mathrm{l}$ for $\mathrm{VnfK}$ detection and $15 \mu \mathrm{l}$ for $\mathrm{VnfG}$ detection. After SDSPAGE, proteins were transferred to nitrocellulose membranes and detected with specific antibodies against NifDK, MosAB, $\mathrm{VnfK}$, or VnfG used at 1:2500 dilutions. MosAB polyclonal antibodies were produced in rabbit (CIB-CSIC). Secondary anti-rabbit Alkaline Phosphatase was used at 1:5000 dilution. NBT/BCIP (Nitroblue tetrazolium and 5-Bromo-4-chloro-3indolyl phosphate) was used to develop immunodetection signal membranes. Image software was used to quantify the protein levels in immunoblot membranes. The amount of MoSto protein in partially purified preparations from A. vinelandii cells was quantified against calibration curves generated with known amounts of pure MoSto obtained from recombinant $E$. coli cells.

\section{Purification of MoSto From A. vinelandii and Recombinant E. coli Cells}

Escherichia coli BL21(DE3) pLysS pN2MN72 cell-free extracts were prepared by mixing cells in binding buffer $50 \mathrm{mM} \mathrm{Na}_{3} \mathrm{PO}_{4}$ pH 7.2 buffer, $500 \mathrm{mM} \mathrm{NaCl}, 10 \mathrm{mM}$ imidazole in a ratio 1:2 and passing twice through a French Press (1,500 psi) followed by ultracentrifugation at 24,000 r.p.m. for $30 \mathrm{~min}$ at $4^{\circ} \mathrm{C}$. Cell free extract is loaded into HiTrap $\mathrm{Ni}^{+}$column (GE Healthcare) pre-equilibrated with binding buffer in a AKTA prime FPLC inside the glove box. The column was washed with binding buffer followed by two extra washes at 30 and $60 \mathrm{mM}$ imidazole. Elution was carried out at $250 \mathrm{mM}$ of imidazole. Eluted fractions containing purified MoSto were pooled, desalted and exchanged in $50 \mathrm{mM} \mathrm{Na}_{3} \mathrm{PO}_{4} \mathrm{pH} 7.2$ buffer, $500 \mathrm{mM} \mathrm{NaCl}, 10 \%$ glycerol. MoSto was stored in liquid nitrogen until used.

To purify MoSto from $A$. vinelandii cells, strain DJ was grown in 100-L fermentor with Burk's modified medium containing $10 \mu \mathrm{MNa}_{2} \mathrm{MoO}_{4}$ and $12.8 \mathrm{mM}$ urea at $30^{\circ} \mathrm{C}$ for $20 \mathrm{~h}$ maintaining $3 \%$ dissolved $\mathrm{O}_{2}$. Cell-free extract preparations (French Press followed by cell debris removal by ultracentrifugation) and MoSto purification, including DEAESephacel chromatography, ammonium sulfate fractionation, and Superdex-200 gel filtration were performed as described (Fenske et al., 2005). Fractions pooled and used for further chromatographic steps were selected according to their SDSPAGE profiles and Mo determination results. MoSto was stored in liquid nitrogen until used.

\section{REFERENCES}

Allen, R. M., Roll, J. T., Rangaraj, P., Shah, V. K., Roberts, G. P., and Ludden, P. W. (1999). Incorporation of molybdenum into the iron-molybdenum cofactor of nitrogenase. J. Biol. Chem. 274, 15869-15874. doi: 10.1074/jbc.274.22.15869

Allen, R. S., Tilbrook, K., Warden, A. C., Campbell, P. C., Rolland, V., Singh, S. P., et al. (2017). Expression of 16 nitrogenase proteins within the plant mitochondrial matrix. Front. Plant Sci. 8:287. doi: 10.3389/fpls.2017. 00287

\section{In vitro FeMo-co Synthesis and Nitrogenase Activation Assays}

In vitro FeMo-co synthesis assays were carried out as described (Curatti et al., 2007). Reactions were carried out in acid-treated 9$\mathrm{ml}$ serum vials sealed with serum stoppers under argon/acetylene (93\%/7\%) atmosphere. When indicated, MoSto purified either from E. coli or from A. vinelandii was added to the in vitro FeMo-co synthesis reactions as sole source of Mo. Activity of reconstituted NifDK was analyzed by the acetylene reduction assay after addition of excess NifH as described (Curatti et al., 2007). Ethylene formation was measured in a Shimadzu GC-2014 gas chromatograph equipped with a PoraPak N 80-100 column. The specific activity of each protein is defined as nanomoles of ethylene formed per minute per mg of NifDK protein.

Protein-protein interaction requirement for Mo donation by MoSto was addressed by inserting a $3-\mathrm{kDa}$ pore-size cutoff dialysis membrane between a MoSto solution on one side and the mixture of FeMo-co biosynthetic proteins and apoNifDK on the other side. FeMo-co synthesis and nitrogenase activation reactions were carried out at $30^{\circ} \mathrm{C}$. Samples to quantify reconstituted nitrogenase activity were taken at 2,10 , and $30 \mathrm{~min}$ and analyzed for acetylene reduction.

\section{AUTHOR CONTRIBUTIONS}

MN-R and JB carried out the experimental work. MN-R and LR performed experimental design, data analysis, and wrote the manuscript.

\section{FUNDING}

Funding for this research was provided by the Bill \& Melinda Gates Foundation Grant OPP1143172 (to LR) and UPM grant RP160050022 (to LR). MN-R was supported by a contract of the Programa Operativo de Empleo Juvenil y la Iniciativa de Empleo Juvenil (YEI) with the participation of the Consejeriia de Educacioin, Juventud y Deporte de la Comunidad de Madrid and the European Social Fund.

\section{ACKNOWLEDGMENTS}

We thank Zinaida Perova for the construction of plasmid pRHB268.

Bishop, P. E., and Joerger, R. D. (1990). Genetics and molecular biology of alternative nitrogen fixation systems. Annu. Rev. Plant Physiol. Plant Mol. Biol. 41, 109-125. doi: 10.1146/annurev.pp.41.060190.000545

Boyd, E. S., and Peters, J. W. (2013). New insights into the evolutionary history of biological nitrogen fixation. Front. Microbiol. 4:201. doi: 10.3389/fmicb.2013. 00201

Brandner, J. P., McEwan, A. G., Kaplan, S., and Donohue, T. J. (1989). Expression of the Rhodobacter sphaeroides cytochrome c2 structural gene. J. Bacteriol. 171, 360-368. doi: 10.1128/jb.171.1.360-368.1989 
Buren, S., and Rubio, L. M. (2018). State of the art in eukaryotic nitrogenase engineering. FEMS Microbiol. Lett. 365:fnx274. doi: 10.1093/femsle/ fnx274

Buren, S., Young, E. M., Sweeny, E. A., Lopez-Torrejon, G., Veldhuizen, M., Voigt, C. A., et al. (2017). Formation of nitrogenase NifDK tetramers in the mitochondria of Saccharomyces cerevisiae. ACS Synth. Biol. 6, 1043-1055. doi: 10.1021/acssynbio.6b00371

Cárdenas, J., and Mortenson, L. E. (1974). Determination of molybdenum and tungsten in biological materials. Anal. Biochem. 60, 372-381. doi: 10.1016/00032697(74)90244-9

Chatterjee, R., Allen, R. M., Shah, V. K., and Ludden, P. W. (1994). Nucleotide and divalent cation specificity of in vitro iron-molybdenum cofactor synthesis. J. Bacteriol. 176, 2747-2750. doi: 10.1128/jb.176.9.2747-2750.1994

Chatterjee, R., Ludden, P. W., and Shah, V. K. (1997). Characterization of VNFG, the delta subunit of the $v n f$-encoded apodinitrogenase from Azotobacter vinelandii. Implications for its role in the formation of functional dinitrogenase 2. J. Biol. Chem. 272, 3758-3765. doi: 10.1074/jbc.272.6.3758

Curatti, L., Brown, C. S., Ludden, P. W., and Rubio, L. M. (2005). Genes required for rapid expression of nitrogenase activity in Azotobacter vinelandii. Proc. Natl. Acad. Sci. U.S.A. 102, 6291-6296. doi: 10.1073/pnas.0501216102

Curatti, L., Hernandez, J. A., Igarashi, R. Y., Soboh, B., Zhao, D., and Rubio, L. M. (2007). In vitro synthesis of the iron-molybdenum cofactor of nitrogenase from iron, sulfur, molybdenum and homocitrate using purified proteins. Proc. Natl. Acad. Sci. U.S.A. 104, 17626-17631. doi: 10.1073/pnas.0703050104

Dilworth, M. J., Eady, R. R., and Eldridge, M. E. (1988). The vanadium nitrogenase of Azotobacter chroococcum: reduction of acetylene and ethylene to ethane. Biochem. J. 249, 745-751. doi: 10.1042/bj2490745

Dilworth, M. J., Eady, R. R., Robson, R. L., and Miller, R. W. (1987). Ethane formation from acetylene as a potential test for vanadium nitrogenase in vivo. Nature 327, 167-168. doi: 10.1038/327167a0

Dos Santos, P. C. (2011). Molecular biology and genetic engineering in nitrogen fixation. Methods Mol. Biol. 766, 81-92. doi: 10.1007/978-1-61779-194-9_6

Eady, R. R. (1996). Structure-function relationships of alternative nitrogenases. Chem. Rev. 96, 3013-3030. doi: 10.1021/cr950057h

Einsle, O., Tezcan, F. A., Andrade, S. L., Schmid, B., Yoshida, M., Howard, J. B., et al. (2002). Nitrogenase MoFe-protein at 1.16 A resolution: a central ligand in the FeMo-cofactor. Science 297, 1696-1700. doi: 10.1126/science.1073877

Fenske, D., Gnida, M., Schneider, K., Meyer-Klaucke, W., Schemberg, J., Henschel, V., et al. (2005). A new type of metalloprotein: the Mo storage protein from Azotobacter vinelandii contains a polynuclear molybdenum-oxide cluster. Chembiochem 6, 405-413. doi: 10.1002/cbic.200400263

George, S. J., Igarashi, R. Y., Piamonteze, C., Soboh, B., Cramer, S. P., and Rubio, L. M. (2007). Identification of a Mo-Fe-S cluster on NifEN by Mo K-edge extended X-ray absorption fine structure. J. Am. Chem. Soc. 129, 3060-3061. doi: $10.1021 /$ ja0663428

Georgiadis, M. M., Komiya, H., Chakrabarti, P., Woo, D., Kornuc, J. J., and Rees, D. C. (1992). Crystallographic structure of the nitrogenase iron protein from Azotobacter vinelandii. Science 257, 1653-1659. doi: 10.1126/science.1529353

Hernandez, J. A., Curatti, L., Aznar, C. P., Perova, Z., Britt, R. D., and Rubio, L. M. (2008). Metal trafficking for nitrogen fixation: NifQ donates molybdenum to NifEN/NifH for the biosynthesis of the nitrogenase FeMo-cofactor. Proc. Natl. Acad. Sci. U.S.A. 105, 11679-11684. doi: 10.1073/pnas.0803576105

Hernandez, J. A., George, S. J., and Rubio, L. M. (2009). Molybdenum trafficking for nitrogen fixation. Biochemistry 48, 9711-9721. doi: 10.1021/bi901217p

Hille, R., Hall, J., and Basu, P. (2014). The mononuclear molybdenum enzymes. Chem. Rev. 114, 3963-4038. doi: 10.1021/cr400443z

Jacobitz, S., and Bishop, P. E. (1992). Regulation of nitrogenase-2 in Azotobacter vinelandii by ammonium, molybdenum, and vanadium. J. Bacteriol. 174, 3884-3888. doi: 10.1128/jb.174.12.3884-3888.1992

Jacobson, M. R., Premakumar, R., and Bishop, P. E. (1986). Transcriptional regulation of nitrogen fixation by molybdenum in Azotobacter vinelandii. J. Bacteriol. 167, 480-486. doi: 10.1128/jb.167.2.480486.1986

Joerger, R. D., and Bishop, P. E. (1988). Nucleotide sequence and genetic analysis of the nifB-nifQ region from Azotobacter vinelandii. J. Bacteriol. 170, 1475-1487. doi: 10.1128/jb.170.4.1475-1487.1988

Joerger, R. D., Loveless, T. M., Pau, R. N., Mitchenall, L. A., Simon, B. H., and Bishop, P. E. (1990). Nucleotide sequences and mutational analysis of the structural genes for nitrogenase 2 of Azotobacter vinelandii. J. Bacteriol. 172, 3400-3408. doi: 10.1128/jb.172.6.3400-3408.1990

Keeler, R. F., and Varner, J. E. (1957). Tungstate as an antagonist of molybdate in Azotobacter vinelandii. Arch. Biochem. Biophys. 70, 585-590. doi: 10.1016/00039861(57)90146-7

Kim, J., and Rees, D. C. (1992). Crystallographic structure and functional implications of the nitrogenase molybdenum-iron protein from Azotobacter vinelandii. Nature 360, 553-560. doi: 10.1038/360553a0

Kowalewski, B., Poppe, J., Demmer, U., Warkentin, E., Dierks, T., Ermler, U., et al. (2012). Nature's polyoxometalate chemistry: X-ray structure of the Mo storage protein loaded with discrete polynuclear Mo-O clusters. J. Am. Chem. Soc. 134, 9768-9774. doi: 10.1021/ja303084n

Kraepiel, A. M., Bellenger, J. P., Wichard, T., and Morel, F. M. (2009). Multiple roles of siderophores in free-living nitrogen-fixing bacteria. Biometals 22, 573-581. doi: 10.1007/s10534-009-9222-7

Krahn, E., Weiss, R., Krockel, M., Groppe, J., Henkel, G., Cramer, P., et al. (2002). The Fe-only nitrogenase from Rhodobacter capsulatus: identification of the cofactor, an unusual, high-nuclearity iron-sulfur cluster, by Fe K-edge EXAFS and 57Fe Mossbauer spectroscopy. J. Biol. Inorg. Chem. 7, 37-45. doi: 10.1007/ s007750100263

Laemmli, U. K. (1970). Cleavage of structural proteins during the assembly of the head of bacteriophage T4. Nature 227, 680-685. doi: 10.1038/227680a0

Luque, F., and Pau, R. N. (1991). Transcriptional regulation by metals of structural genes for Azotobacter vinelandii nitrogenases. Mol. Gen. Genet. 227, 481-487. doi: 10.1007/BF00273941

Macho, A. P., Zumaquero, A., Ortiz-Martin, I., and Beuzon, C. R. (2007). Competitive index in mixed infections: a sensitive and accurate assay for the genetic analysis of Pseudomonas syringae-plant interactions. Mol. Plant Pathol. 8, 437-450. doi: 10.1111/j.1364-3703.2007.00404.x

Moore, V. G., Tittsworth, R. C., and Hales, B. J. (1994). Construction and characterization of hybrid component 1 from V-nitrogenase containing FeMo cofactor. J. Am. Chem. Soc. 116, 12101-12102. doi: 10.1021/ja00105a080

Mouncey, N. J., Mitchenall, L. A., and Pau, R. N. (1995). Mutational analysis of genes of the mod locus involved in molybdenum transport, homeostasis, and processing in Azotobacter vinelandii. J. Bacteriol. 177, 5294-5302. doi: 10.1128/ jb.177.18.5294-5302.1995

Obranic, S., Babic, F., and Maravic-Vlahovicek, G. (2013). Improvement of pBBR1MCS plasmids, a very useful series of broad-host-range cloning vectors. Plasmid 70, 263-267. doi: 10.1016/j.plasmid.2013.04.001

Pienkos, P. T., and Brill, W. J. (1981). Molybdenum accumulation and storage in Klebsiella pneumoniae and Azotobacter vinelandii. J. Bacteriol. 145, 743-751.

Pinske, C., Bonn, M., Kruger, S., Lindenstrauss, U., and Sawers, R. G. (2011). Metabolic deficiences revealed in the biotechnologically important model bacterium Escherichia coli BL21(DE3). PLoS One 6:e22830. doi: 10.1371/ journal.pone.0022830

Poppe, J., Brunle, S., Hail, R., Wiesemann, K., Schneider, K., and Ermler, U. (2018). The molybdenum storage protein: a soluble ATP hydrolysis-dependent molybdate pump. FEBS J. 285, 4602-4616. doi: 10.1111/febs.14684

Poppe, J., Warkentin, E., Demmer, U., Kowalewski, B., Dierks, T., Schneider, K., et al. (2014). Structural diversity of polyoxomolybdate clusters along the threefold axis of the molybdenum storage protein. J. Inorg. Biochem. 138, 122-128. doi: 10.1016/j.jinorgbio.2014.05.009

Poza-Carrion, C., Jimenez-Vicente, E., Navarro-Rodriguez, M., EchavarriErasun, C., and Rubio, L. M. (2014). Kinetics of nif gene expression in a nitrogen-fixing bacterium. J. Bacteriol. 196, 595-603. doi: 10.1128/jb.00942-13

Prentki, P., and Krisch, H. M. (1984). In vitro insertional mutagenesis with a selectable DNA fragment. Gene 29, 303-313. doi: 10.1016/0378-1119(84)90059-3

Ramon-Maiques, S., Marina, A., Gil-Ortiz, F., Fita, I., and Rubio, V. (2002). Structure of acetylglutamate kinase, a key enzyme for arginine biosynthesis and a prototype for the amino acid kinase enzyme family, during catalysis. Structure 10, 329-342. doi: 10.1016/S0969-2126(02)00721-9

Reddy, K. J., Munn, L. C., and Wang, L. (1997). "Chemistry and mineralogy of molybdenum in soils," in Molybdenum in Agriculture, ed. U. C. Gupta (Cambridge: Cambridge University Press).

Robinson, A. C., Burgess, B. K., and Dean, D. R. (1986). Activity, reconstitution, and accumulation of nitrogenase components in Azotobacter vinelandii mutant strains containing defined deletions within the nitrogenase structural 
gene cluster. J. Bacteriol. 166, 180-186. doi: 10.1128/jb.166.1.180-186. 1986

Rodriguez-Quinones, F., Bosch, R., and Imperial, J. (1993). Expression of the nifBfdxNnifOQ region of Azotobacter vinelandii and its role in nitrogenase activity. J. Bacteriol. 175, 2926-2935. doi: 10.1128/jb.175.10.2926-2935. 1993

Rubio, L. M., and Ludden, P. W. (2008). Biosynthesis of the iron-molybdenum cofactor of nitrogenase. Annu. Rev. Microbiol. 62, 93-111. doi: 10.1146/ annurev.micro.62.081307.162737

Rubio, L. M., Singer, S. W., and Ludden, P. W. (2004). Purification and characterization of NafY (apodinitrogenase gamma subunit) from Azotobacter vinelandii. J. Biol. Chem. 279, 19739-19746. doi: 10.1074/jbc.M400965200

Sambrook, J., and Russell, D. W. (2001). Molecular Cloning. A Laboratory Manual. Cold Spring Harbor, NY: Cold Spring Harbor Laboratory Press.

Schemberg, J., Schneider, K., Demmer, U., Warkentin, E., Muller, A., and Ermler, U. (2007). Towards biological supramolecular chemistry: a variety of pockettemplated, individual metal oxide cluster nucleations in the cavity of a $\mathrm{Mo} / \mathrm{W}$ storage protein. Angew. Chem. Int. Ed. Engl. 46, 2408-2413. doi: 10.1002/anie. 200604858

Schemberg, J., Schneider, K., Fenske, D., and Muller, A. (2008). Azotobacter vinelandii metal storage protein: "classical" inorganic chemistry involved in Mo/W uptake and release processes. Chembiochem 9, 595-602. doi: 10.1002/ cbic. 200700446

Setubal, J. C., dos Santos, P., Goldman, B. S., Ertesvåg, H., Espin, G., Rubio, L. M., et al. (2009). Genome sequence of Azotobacter vinelandii, an obligate aerobe specialized to support diverse anaerobic metabolic processes. J. Bacteriol. 191, 4534-4545. doi: 10.1128/JB.00504-09

Shah, V. K., Ugalde, R. A., Imperial, J., and Brill, W. J. (1984). Molybdenum in nitrogenase. Annu. Rev. Biochem. 53, 231-257. doi: 10.1146/annurev.bi.53. 070184.001311

Sippel, D., and Einsle, O. (2017). The structure of vanadium nitrogenase reveals an unusual bridging ligand. Nat. Chem. Biol. 13, 956-960. doi: 10.1038/nchembio. 2428

Smith, P. K., Krohn, R. I., Hermanson, G. T., Mallia, A. K., Gartner, F. H., Provenzano, M. D., et al. (1985). Measurement of protein using bicinchoninic acid. Anal. Biochem. 150, 76-85. doi: 10.1016/0003-2697(85)90442-7
Soboh, B., Boyd, E. S., Zhao, D., Peters, J. W., and Rubio, L. M. (2010). Substrate specificity and evolutionary implications of a NifDK enzyme carrying NifB-co at its active site. FEBS Lett. 584, 1487-1492. doi: 10.1016/j.febslet.2010.02.064

Soboh, B., Igarashi, R. Y., Hernandez, J. A., and Rubio, L. M. (2006). Purification of a NifEN protein complex that contains bound molybdenum and a FeMo-Co precursor from an Azotobacter vinelandii DeltanifHDK strain. J. Biol. Chem. 281, 36701-36709. doi: 10.1074/jbc.M6068 20200

Spatzal, T., Aksoyoglu, M., Zhang, L., Andrade, S. L., Schleicher, E., Weber, S., et al. (2011). Evidence for interstitial carbon in nitrogenase FeMo cofactor. Science 334:940. doi: 10.1126/science. 1214025

Stewart, W. P. D., Fitzgerald, G. P., and Burris, R. H. (1967). In situ studies on N2 fixation using the acetylene reduction technique. Proc. Natl. Acad. Sci. U.S.A. 58, 2071-2078. doi: 10.1073/pnas.58. 5.2071

Strandberg, G. W., and Wilson, P. W. (1968). Formation of the nitrogen-fixing enzyme system in Azotobacter vinelandii. Can. J. Microbiol. 14, 25-31. doi: $10.1139 / \mathrm{m} 68-005$

Studier, F. W., and Moffatt, B. A. (1986). Use of bacteriophage T7 RNA polymerase to direct selective high-level expression of cloned genes. J. Mol. Biol. 189, 113-130. doi: 10.1016/0022-2836(86)90385-2

Wichard, T., Bellenger, J. P., Loison, A., and Kraepiel, A. M. (2008). Catechol siderophores control tungsten uptake and toxicity in the nitrogen-fixing bacterium Azotobacter vinelandii. Environ. Sci. Technol. 42, 2408-2413. doi: $10.1021 /$ es702651f

Conflict of Interest Statement: The authors declare that the research was conducted in the absence of any commercial or financial relationships that could be construed as a potential conflict of interest.

Copyright (C) 2019 Navarro-Rodríguez, Buesa and Rubio. This is an open-access article distributed under the terms of the Creative Commons Attribution License (CC BY). The use, distribution or reproduction in other forums is permitted, provided the original author(s) and the copyright owner(s) are credited and that the original publication in this journal is cited, in accordance with accepted academic practice. No use, distribution or reproduction is permitted which does not comply with these terms. 